\title{
Seawater-driven magnesium based Janus micromotors for environmental remediation $\uparrow$
}

Cite this: Nanoscale, 2013, 5, 4696

\author{
Wei Gao, Xiaomiao Feng, Allen Pei, + Yonge Gu, Jinxing Li and Joseph Wang*
}

Received 25th March 2013

Accepted 25th April 2013

DOI: $10.1039 / c 3 n r 01458 d$

www.rsc.org/nanoscale

We describe the use of seawater as fuel to propel Janus micromotors. The new micromotors consist of biodegradable and environmentally friendly magnesium microparticles and a nickel-gold bilayer patch for magnetic guidance and surface modification. Such seawaterdriven micromotors, which utilize macrogalvanic corrosion and chloride pitting corrosion processes, eliminate the need for external fuels to offer efficient and prolonged propulsion towards diverse applications in aquatic environments.

The propulsion of synthetic nanoscale objects represents a great challenge and opportunity, and has thus stimulated considerable research efforts in recent years. ${ }^{1-10}$ Self-propelled micromotors offer promise for diverse practical applications, such as drug delivery, ${ }^{\mathbf{1 1 , 1 2}}$ biomaterials isolation, ${ }^{13-15}$ surface patterning $^{\mathbf{1 6}}$ and environmental remediation. ${ }^{17}$ Bubblepropelled catalytic micromotors are particularly attractive due to their efficient propulsion in relevant biological fluids and high ionic-strength media. ${ }^{18-21}$ Unfortunately, the requirement of the hydrogen peroxide fuel greatly impedes many practical applications of such catalytic micro/nanoscale motors. ${ }^{7}$ New micro/nanomotors that can harvest energy from their own surrounding environment, i.e., use the sample matrix itself as their fuel source, are highly desired for eliminating the need for adding external fuels. For example, Gao et al. illustrated hydrogen-bubble-propelled micromotors that can be powered in acidic or alkaline media. ${ }^{22,23}$ However, water is the obvious ideal choice of fuel for the majority of practical nanomachine applications compared to extreme acidic or alkaline media. Recently we described the first example of a water-driven micromotor, based on $\mathrm{Al} / \mathrm{Ga}$ microparticles, which displayed efficient propulsion in aqueous solutions. ${ }^{24}$ However, due to the

Department of Nanoengineering, University of California, San Diego, La Jolla, California 92093, USA. E-mail: josephwang@ucsd.edu

† Electronic supplementary information (ESI) available: Supporting videos. See DOI: $10.1039 / \mathrm{c} 3$ nr01458d

\$ These authors contributed equally to this work. toxicity of aluminum and gallium, more biocompatible and environmentally friendly materials are highly desired for different applications of water-driven micromotors.

Here we demonstrate a new hydrogen-bubble-propelled Janus micromotor, based on the magnesium-water reaction, which can be self-propelled in seawater without an external fuel. Common active metals (e.g., Li, Na, K, Ca), can lead to efficient hydrogen evolution from the water-metal reaction, but are too reactive for a safe operation and are not stable in air. Magnesium, in contrast, is an attractive candidate material for the design of water-driven micromotors as it is a biocompatible 'green' nutrient trace element, vital for many bodily functions and enzymatic processes. In addition, $\mathrm{Mg}$ is a low cost metal and $\mathrm{Mg}^{2+}$ is present in different natural environments (e.g., seawater). The $\mathrm{Mg}$-water reaction is commonly hindered in ambient atmospheres due to the formation of a compact hydroxide passivation surface layer. Accordingly, $\mathrm{Mg}$ cannot continuously reduce water to generate hydrogen bubbles. ${ }^{25,26}$ However, we demonstrate in the following sections that the micromotor can display efficient and prolonged propulsion in chloride-rich environments (such as seawater) owing to the combination of macrogalvanic corrosion and chloride pitting corrosion processes (discussed below). The new seawater-driven micromotors can be guided magnetically (through the incorporation of a magnetic $\mathrm{Ni}$ layer) and be functionalized to perform various important tasks. For example, modification of the outer gold patch with long-chain alkanethiols leads to a super-hydrophobic surface that can be used for environmental oil remediation.

Fig. 1 displays the schematic of the hydrogen-bubblepropelled seawater-driven Janus Mg-based micromotors. The $\mathrm{Mg}$ particles have an average size of $\sim 30 \mu \mathrm{m}$ and are asymmetrically coated by e-beam evaporation with $\mathrm{Ti}, \mathrm{Ni}$ and $\mathrm{Au}$ layers to form the Janus micromotors (with the $\mathrm{Ti}$ layer providing a good contact between the $\mathrm{Ni}-\mathrm{Au}$ layers and the $\mathrm{Mg}$ surface). Upon immersion into seawater, a spontaneous redox reaction occurs, involving the oxidation of the $\mathrm{Mg}$ surface to reduce water to hydrogen bubbles: 


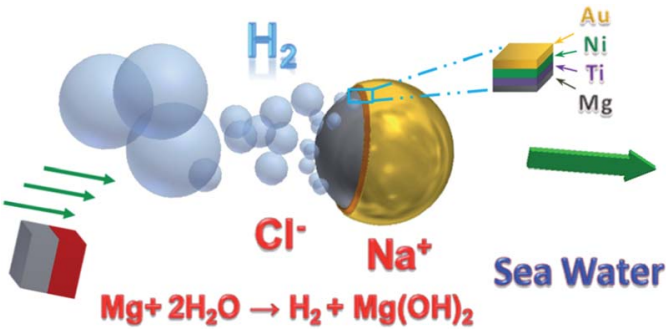

Fig. 1 Schematic of the Mg-based seawater-driven Janus micromotors.

$$
\mathrm{Mg}(\mathrm{s})+2 \mathrm{H}_{2} \mathrm{O}(\mathrm{aq}) \rightarrow \mathrm{Mg}(\mathrm{OH})_{2}(\mathrm{~s})+\mathrm{H}_{2}(\mathrm{~g})
$$

While the rapidly formed oxide passivation layer on the $\mathrm{Mg}$ surface can hinder the process, ${ }^{25,26}$ we found that the presence of the gold layer and chloride ions allows the reaction to proceed (by combination of macrogalvanic corrosion and pitting corrosion processes, respectively), hence leading to continuous formation of hydrogen bubbles to propel the microparticles.

Fig. 2 displays time-lapse images, taken from ESI Video $1, \dagger$ of the movement of the Janus $\mathrm{Mg}$ micromotor in seawater over a $4 \mathrm{~s}$ period at $1 \mathrm{~s}$ intervals. These images illustrate the continuous stream of hydrogen microbubbles released from the exposed $\mathrm{Mg}$ surface. The micromotor is self-propelled at a high speed of over $90 \mu \mathrm{m} \mathrm{s}^{-1}$, which corresponds to a relative speed of nearly 3 body lengths per $\mathrm{s}$. The $\mathrm{Mg}$ Janus micromotor follows predetermined trajectories under the magnetic guidance. As illustrated in Fig. 2b-e and ESI Video $1, \dagger$ such magnetic guidance steers the micromotor along different trajectories to offer high spatial and temporal resolution essential for a variety of applications. The micromotor thus executes sharp and rapid turns and travels in a square pattern.

The presence of the gold layer plays a crucial role in the selfpropulsion of the $\mathrm{Mg}$-based micromotor, as was demonstrated by various control experiments. Fig. 3A and corresponding ESI Video $2 \uparrow$ clearly indicate that the bare $\mathrm{Mg}$ particles and $\mathrm{Mg}-\mathrm{Ti}$ Janus particles display no bubble generation and net displacement in seawater. Some bubble formation, with no directional propulsion, is observed using the $\mathrm{Mg}-\mathrm{Ti}-\mathrm{Ag}$ microparticle in the seawater. In contrast, replacing the Ag layer with a Au layer leads to efficient motion in seawater, reflecting the enhanced $\mathrm{Mg}^{-}$ water reaction. The important role of the gold in the increased reaction rate and propulsion efficiency is associated with the

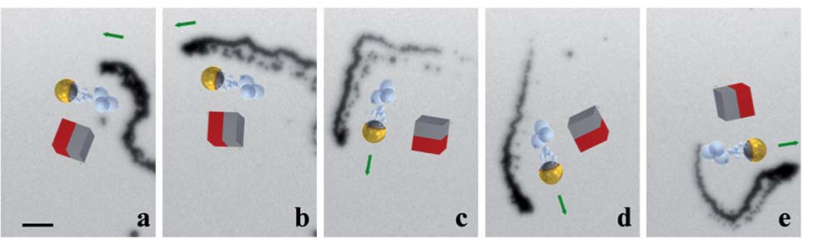

Fig. 2 Seawater-driven magnetically guided Mg-based micromotor: time-lapse images showing the propulsion of micromotor in seawater in one second intervals with spatial magnetic motion control. Scale bar, $40 \mu \mathrm{m}$.

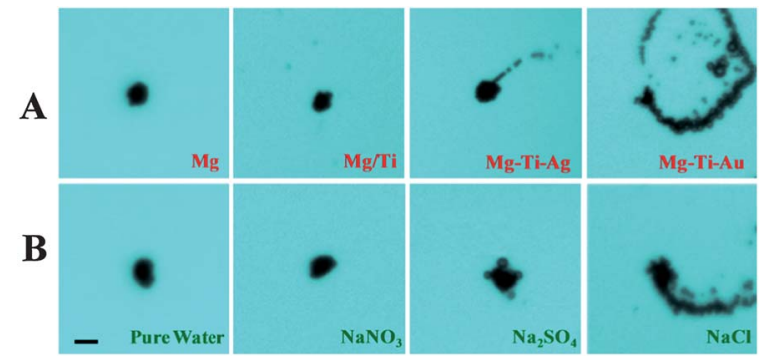

Fig. 3 (A) Control experiments using $\mathrm{Mg}, \mathrm{Mg}-\mathrm{Ti}, \mathrm{Mg}-\mathrm{Ti}-\mathrm{Ag}$ and $\mathrm{Mg}-\mathrm{Ti}-\mathrm{Au}$ microparticles in seawater over a 5 second period. (B) Anion effect upon the movement of $\mathrm{Mg}-\mathrm{Ti}-\mathrm{Au}$ micromotors using pure water, $0.5 \mathrm{M} \mathrm{NaNO}_{3}, 0.5 \mathrm{M}$ $\mathrm{Na}_{2} \mathrm{SO}_{4}$ and $0.5 \mathrm{M} \mathrm{NaCl}$ solutions (over a 6 second period). Scale bar, $30 \mu \mathrm{m}$.

macrogalvanic corrosion mechanism, which is an electrochemical process in which one metal corrodes preferentially to another one when both metals are in electrical contact and immersed in an electrolyte. ${ }^{27}$ When the Janus micromotor is immersed into seawater, a short-circuited galvanic cell is formed. The anodic indexes for gold and silver are $-0.0 \mathrm{~V}$ and $-0.15 \mathrm{~V}$, respectively, while magnesium has a very low potential of $-1.75 \mathrm{~V}^{28}$ The $\mathrm{Au}-\mathrm{Mg}$ system thus has a larger electrochemical potential difference, compared to the $\mathrm{Ag}-\mathrm{Mg}$ couple, resulting in a considerably faster and preferential dissolution of magnesium in the presence of gold.

Additionally, we observed that such macrogalvanic corrosion of $\mathrm{Mg}$ (and the corresponding propulsion) is strongly influenced by co-existing anions. For example, Fig. 3B and ESI Video $3 \uparrow$ compare the bubble generation and motion of $\mathrm{Mg}-\mathrm{Ti}-\mathrm{Au}$ Janus micromotors in the presence of different electrolytes $\left(\mathrm{NaNO}_{3}, \mathrm{Na}_{2} \mathrm{SO}_{4}\right.$, and $\left.\mathrm{NaCl}\right)$. No bubble generation is observed on the micromotors in pure water and sodium nitrate solutions, while very slow bubble generation is observed in the sodium sulfate medium; in contrast, a fast and strong bubble generation is observed in the sodium chloride solution. Overall, Fig. 3B clearly indicates that the chloride-induced pitting corrosion on the $\mathrm{Mg}$ surface plays a critical role in the observed bubble generation and propulsion (in addition to the galvanic corrosion). Pitting corrosion commonly occurs in metals that are protected by a passivation layer (e.g., $\mathrm{Mg}$ with a $\mathrm{Mg}(\mathrm{OH})_{2}$ layer) ${ }^{29-32}$ It is well known that chloride ions promote the corrosion of $\mathrm{Mg}$ in aqueous solutions. ${ }^{31}$ Aggressive anionic species, such as chloride, are able to penetrate the passivation layer and are further electrostatically transported into the pit, where they serve to balance the charge within the corrosion pits as the $\mathrm{Mg}^{+}$cation concentration builds up..$^{27,29}$ As the $\mathrm{Mg}$ corrosion continues, the $\mathrm{OH}^{-}$is depleted within the pit, preventing the passivation of the pit surfaces. ${ }^{30}$ The pit environment becomes weakly acidic due to the build-up of $\mathrm{Mg}^{+}$and $\mathrm{Cl}^{-}$ levels, further promoting the $\mathrm{Mg}$ dissolution in the pit. The intermediate $\mathrm{Mg}^{+}$near the surface of the pit then reacts to form $\mathrm{H}_{2}$ gas: $^{29}$

$$
2 \mathrm{Mg}^{+}(\mathrm{aq})+2 \mathrm{H}_{2} \mathrm{O}(\mathrm{aq}) \rightarrow 2 \mathrm{Mg}^{2+}(\mathrm{aq})+2 \mathrm{OH}^{-}(\mathrm{aq})+\mathrm{H}_{2}(\mathrm{~g})
$$

This process is autocatalytic, and proceeds as long as there is consistent electrolytic migration of $\mathrm{Cl}^{-}$into the pit. ${ }^{30}$ The high 
level of $\mathrm{Cl}^{-}$in seawater $\left(546 \mathrm{mmol} \mathrm{kg}^{-1}\right.$ ) thus strongly promotes this reaction mechanism. Sulfate ions can still facilitate the reaction but display a much weaker effect (compared to the chloride ions), ${ }^{31,32}$ which is consistent with the experimental results shown in Fig. 3B; nitrate ions, in contrast, have no apparent effects on the reaction rate,,$^{31,32}$ and hence no bubbles are observed in $\mathrm{NaNO}_{3}$.

It appears that the macrogalvanic corrosion and pitting corrosion effects - when coupled together as in these $\mathrm{Mg}$ motors - work synergistically to enhance the overall rate of $\mathrm{Mg}$ dissolution and reaction. The galvanic coupling of the $\mathrm{Mg}$ particle with the Au layer leads to an enhanced preferential corrosion and anodic dissolution of $\mathrm{Mg}$ at the exposed regions, along with the cathodic evolution of $\mathrm{H}_{2}$ gas at the Au surface. However, the control experiments clearly illustrate that the $\mathrm{H}_{2}$ bubbles, generated at the $\mathrm{Mg}$ surface in the presence of chloride ions due to the pitting corrosion mechanism, have a dominating effect upon the observed propulsion. In fact, the intrinsic galvanic cell (macrogalvanic and microgalvanic effects from the gold coating and impurities of the $\mathrm{Mg}$ particles, respectively) serves to further increase the anodic dissolution of $\mathrm{Mg}$, assisting in the pitting corrosion. ${ }^{27}$ Thus, the rapidly evolving hydrogen bubbles at the $\mathrm{Mg}$ surface provide the thrust essential for the directional propulsion of the Janus micromotors.

The autonomous motion performance of the new Mg-based Janus micromotors depends strongly upon the chloride ion concentrations in the aqueous media (Fig. 4 and ESI Video $4 \dagger$ ). As was discussed earlier, the presence of chloride ions increases the amount of dissolved intermediate $\mathrm{Mg}^{+}$that can react to form hydrogen. The reaction of the $\mathrm{Mg}-\mathrm{Ti}-\mathrm{Ni}-\mathrm{Au}$ particles can be observed from the evolution of a clear bubble tail even in the $0.001 \mathrm{M}$ sodium chloride media, reflecting the strong pitting corrosion effect of the chloride ions. As expected, ${ }^{31}$ higher chloride concentrations induce faster reaction rates. For example, the micromotors display an efficient speed of $90 \mu \mathrm{m}$ $\mathrm{s}^{-1}$ in $0.3 \mathrm{M}$ sodium chloride (b), and an even higher speed of $300 \mu \mathrm{m} \mathrm{s}^{-1}$ in $3 \mathrm{M}$ sodium chloride (c). This increased rate of bubble generation in chloride solution further confirms the presence of the pitting corrosion mechanism. Despite the continuous dissolution of the $\mathrm{Mg}$ microparticle, the Janus $\mathrm{Au}-$ Ti-Ni-Mg micromotors display a lifetime of over one min in seawater. Various factors, including the particle size and shape and the exact fuel composition are currently being examined towards extending the motor lifetime.

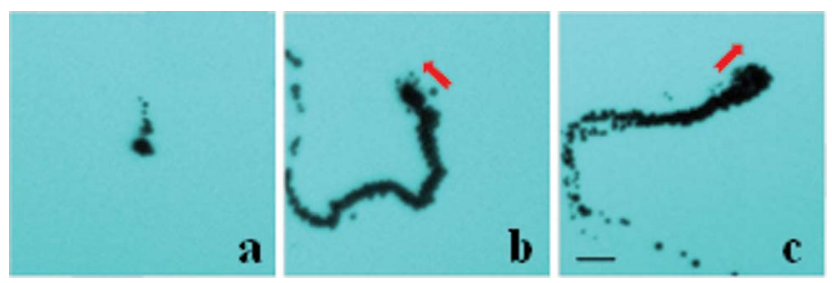

Fig. 4 Motion of the Mg-Ti-Ni-Au micromotors in the presence of different

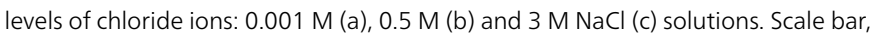
$50 \mu \mathrm{m}$.
We also examined the influence of the solution $\mathrm{pH}$ and the presence of a metal complexing agent (EDTA) upon the speed of the micromotors. The micromotors displayed a comparable propulsion behavior in weak acidic or alkaline seawater of $\mathrm{pH}$ values ranging from $\mathrm{pH} 4$ to $\mathrm{pH} 10$, indicating minimal effect of these media upon the efficiency of the $\mathrm{Mg}$-water reaction. Even faster $\mathrm{Mg}$ dissolutions and propulsions were observed in strongly acidic environments $(\mathrm{pH}<2)$, while strongly alkaline media $(\mathrm{pH}>12)$ resulted in nearly complete hindrance of the Mg-water reaction. ${ }^{27}$ Adding $0.1 \mathrm{mM}$ EDTA to the natural seawater resulted in a decreased speed (to $40 \mu \mathrm{m} \mathrm{s}^{-1}$ ), possibly due to chelation of $\mathrm{Mg}^{+}$ions, and hence hindrance of the hydrogen evolution reaction (eqn (2)).

The practical utility of the new $\mathrm{Mg}$-based seawater-driven Janus micromotors has been illustrated in the present study towards the capture and transport of oil droplets from contaminated seawater. The removal of oil spills from contaminated seawater is of considerable importance for minimizing environmental hazards. Most of the common cleaning methods lack the desired efficiency and are neither cost-effective nor environmentally friendly. Accordingly, the development of effective oil-water separation methods is highly desired. Guix et al. reported recently a motion-based oil cleaning strategy involving hydrophobic surface-oil interaction. ${ }^{\mathbf{1 7}}$ However, the micromotors used were powered by the hydrogen peroxide fuel which is not compatible with large-scale environmental cleanup. The seawater-driven $\mathrm{Mg}$ based Janus micromotor can serve as an attractive platform for environmental remediation of oil contaminants. As demonstrated in the schematic in Fig. 5A, the Mg-based micromotors were modified with self-assembled monolayers (SAMs) of long-chain alkanethiols for imparting the desired strong surface hydrophobicity essential for 'on-the-fly' collection of oil droplets. Fig. 5B and corresponding ESI Video $5 \dagger$ demonstrate a SAMmodified $\mathrm{Mg}$ Janus micromotor being guided (by the external magnetic field) to approach (a), capture (b) and transport (c) the motor oil droplets in seawater. The modified micromotor moves initially at a speed of $90 \mu \mathrm{m} \mathrm{s} \mathrm{s}^{-1}$ (similar to that of bare

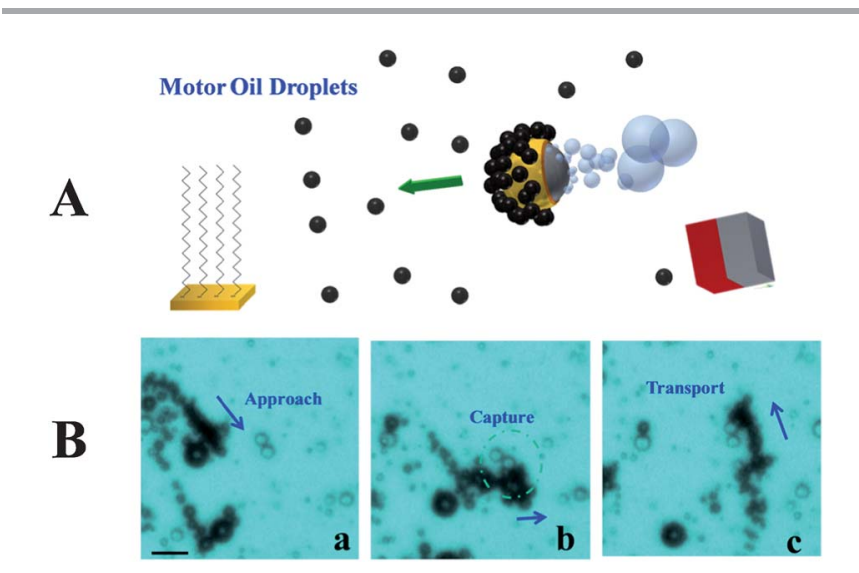

Fig. 5 (A) Schematic hydrophobic seawater driven alkanethiol-modified Mg micromotor for environmental oil remediation. (B) Time lapse images (taken from ESI Video 5 †) of a Mg Janus micromotor approach (a), capture (b) and transport (c) the oil droplet in seawater. Scale bar, $50 \mu \mathrm{m}$. 
micromotors in seawater), indicating that the SAM layer does not affect the galvanic role of the gold layer. Upon contacting the pair of oil droplets (of $20 \mu \mathrm{m}$ diameter each), the micromotor instantaneously captures them. As expected, the speed of the motor greatly decreases to around $44 \mu \mathrm{m} \mathrm{s}^{-1}$ while carrying the droplets, reflecting the larger drag force. In contrast, control experiments involving unmodified micromotors (i.e., without the alkanethiols) displayed no affinity to such droplets after direct contact (ESI Video 6†).

\section{Conclusions}

In conclusion, we have presented a new chemically powered magnesium-based Janus micromotor that propels autonomously using seawater as the sole fuel source and a hydrogen bubble thrust generated from the $\mathrm{Mg}$-water reaction. The hydrogen-bubble propulsion mechanism has been attributed to the combination of galvanic corrosion and pitting corrosion effects, and the important related roles of the gold coating and the ionic (particularly chloride-rich) environments have been discussed. Accordingly, the Mg-based micromotors are expected to move efficiently in other biological media which contain high chloride concentrations. The new water-driven motion capability should greatly expand the scope of applications and environments of chemically powered nanomachines. The composition of the motor and the fuel makes these micromotors highly biocompatible and environmentally friendly. We also demonstrated the first example of a surface-functionalized water-driven micromotor towards practical applications in realistic environments. SAM-modified hydrophobic Mg micromotors were thus applied successfully for oil cleaning in seawater and show considerable potential for environmental remediation. Depending on the specific application, smaller Mg-based motors can be prepared using smaller Mg particles. Additional efforts should be devoted to extending the lifetime of Mg-based motors for addressing the requirements of many practical applications. We expect that the Mg-based Janus micromotor will have a profound impact upon diverse applications in various aqueous environments.

\section{Experimental section}

\section{Synthesis of $\mathrm{Mg}$-Ti-Ni-Au Janus micromotors}

The micromotors were prepared using magnesium microparticles (Catalog \#465666, Sigma-Aldrich, St Louis, MO, powder, -325 mesh, $99.5 \%$ trace metals basis) as the base particles. The $\mathrm{Mg}$ particles were placed onto glass slides and coated with a $20 \mathrm{~nm}$ titanium layer at $1 \AA^{-1}$, a $80 \mathrm{~nm}$ nickel layer at $2 \mathrm{~A} \mathrm{~s}^{-1}$, and a $10 \mathrm{~nm}$ gold layer at $1 \AA^{-1}$ using Temescal BJD 1800 E-beam Evaporator. After a brief sonication in ethanol, the micromotors were released from the glass slide and dispersed into ethanol. The motors were stored in the pure ethanol solution until use. For the control experiments, $\mathrm{Mg}$ particles were similarly coated with $100 \mathrm{~nm} \mathrm{Ti}$ (for $\mathrm{Mg}$-Ti particles), $100 \mathrm{~nm} \mathrm{Ti}$ and $10 \mathrm{~nm} \mathrm{Ag}$ (for Mg-Ti-Ag particles), and $100 \mathrm{~nm} \mathrm{Ti}$ and $10 \mathrm{~nm} \mathrm{Au}$ (for $\mathrm{Mg}-\mathrm{Ti}-\mathrm{Au}$ particles).

\section{Micromotor modification}

The external gold surface of the micromotors was modified by immersion in $1 \mathrm{mM}$ octadecanethiol (Sigma-Aldrich) in absolute ethanol (Sigma-Aldrich) for 1 hour, after which the resulting monolayer-modified micromotors were washed with ethanol and isolated by centrifugation at $6000 \mathrm{rpm}$ for $2 \mathrm{~min}$. All experiments were carried out at room temperature.

\section{Equipment and experimental procedure}

Seawater used as propulsion media in the experiments was collected at Torrey Pines Beach, La Jolla, CA. All the solutions (seawater, $\mathrm{NaCl}, \mathrm{NaNO}_{3}$ and $\mathrm{Na}_{2} \mathrm{SO}_{4}$ ) used in the paper contained $0.5 \%$ Triton X-100 (Fisher Scientific, Fair Lawn, NJ). For the oil remediation experiments, an emulsion of seawatermotor oil sample (20:1), with $0.5 \%$ Triton, was first prepared. Videos were captured by an inverted optical microscope (Nikon Instrument Inc. Ti-S/L100), coupled with $10 \times$ and $4 \times$ objectives, a Hamamatsu digital camera C11440 using the NISElements AR 3.2 software.

\section{Acknowledgements}

This project received support from the Defense Threat Reduction Agency-Joint Science and Technology Office for Chemical and Biological Defense (Grant no. HDTRA1-13-1-0002). W.G. is a HHMI International Student Research fellow. X.M.F. and Y.G. acknowledge financial support from Jiangsu Overseas Research \& Training Program (China) and acknowledge the China Scholarship Council (CSC), respectively. The authors thank J. Orozco and A. Katzenberg for their help.

\section{Notes and references}

1 T. E. Mallouk and A. Sen, Sci. Am., 2009, 300, 72.

2 T. Mirkovic, N. S. Zacharia, G. D. Scholes and G. A. Ozin, ACS Nano, 2010, 4, 1782.

3 J. Wang, ACS Nano, 2009, 3, 4; J. Wang, Nanomachines: Fundamentals and Applications, Wiley-VCH, Weinheim, Germany, 2013, ISBN 978-3-527-33120-8.

4 S. Sanchez and M. Pumera, Chem.-Asian J., 2009, 4, 1402.

5 Y. F. Mei, A. A. Solovev, S. Sanchez and O. G. Schmidt, Chem. Soc. Rev., 2011, 40, 2109.

6 G. Loget and A. Kuhn, J. Am. Chem. Soc., 2010, 132, 15918.

7 J. Wang and W. Gao, ACS Nano, 2012, 6, 5745.

8 M. Pumera, Nanoscale, 2010, 2, 1643.

9 D. Patra, S. Sengupta, W. Duan, H. Zhang, R. Pavlick and A. Sen, Nanoscale, 2013, 5, 1273.

10 G. Loget and A. Kuhn, Nat. Commun., 2011, 2, 535.

11 D. Kagan, R. Laocharoensuk, M. Zimmerman, C. Clawson, S. Balasubramanian, D. Kang, D. Bishop, S. Sattayasamitsathit, L. Zhang and J. Wang, Small, 2010, 6, 2741.

12 W. Gao, D. Kagan, O. S. Pak, C. Clawson, S. Campuzano, E. Chuluun- Erdene, E. Shipton, E. E. Fullerton, L. Zhang, E. Lauga and J. Wang, Small, 2012, 8, 460. 
13 S. Sanchez, A. A. Solovev, S. Schulze and O. G. Schmidt, Chem. Commun., 2011, 47, 698.

14 S. Balasubramanian, D. Kagan, C. M. Hu, S. Campuzano, M. J. Lobo-Castañon, N. Lim, D. Y. Kang, M. Zimmerman, L. Zhang and J. Wang, Angew. Chem., Int. Ed., 2011, 50, 4161. 15 S. Campuzano, J. Orozco, D. Kagan, M. Guix, W. Gao, S. Sattayasamitsathit, J. C. Claussen, A. Merkoçi and J. Wang, Nano Lett., 2012, 12, 396.

16 K. M. Manesh, S. Campuzano, W. Gao, M. J. Lobo-Castañon, I. Shitanda and J. Wang, Nanoscale, 2013, 5, 1310.

17 M. Guix, J. Orozco, M. García, W. Gao, S. Sattayasamitsathit, A. A. Merkoçi, A. Escarpa and J. Wang, ACS Nano, 2012, 6, 4445.

18 Y. F. Mei, G. S. Huang, A. A. Solovev, E. Bermúdez Ureña, I. Mönch, F. Ding, T. Reindl, R. K. Y. Fu, P. K. Chu and O. G. Schmidt, Adv. Mater., 2008, 20, 4085.

19 W. Gao, S. Sattayasamitsathit, J. Orozo and J. Wang, J. Am. Chem. Soc., 2011, 133, 11862.

20 A. A. Solovev, S. Sanchez, M. Pumera, Y. F. Mei and O. G. Schmidt, Adv. Funct. Mater., 2010, 20, 2430.
21 G. Zhao, A. Ambrosi and M. Pumera, Nanoscale, 2013, 5, 1319.

22 W. Gao, A. Uygun and J. Wang, J. Am. Chem. Soc., 2012, 134, 897.

23 W. Gao, M. D'Agostino, V. Garcia-Gradilla, J. Orozco and J. Wang, Small, 2013, 9, 467.

24 W. Gao, A. Pei and J. Wang, ACS Nano, 2012, 6, 8432.

25 G. Lee and J. Park, Geochim. Cosmochim. Acta, 2013, 102, 162.

26 G. L. Makar and J. Kruger, Int. Mater. Rev., 1993, 38, 138.

27 G. Song and A. Atrens, Adv. Eng. Mater., 2003, 5, 837.

28 P. R. Roberge, Handbook of Corrosion Engineering, McGrawHill Professional, New York, 2000.

29 G. Song, A. Atrens, D. St John, X. Wu and J. Nairn, Corros. Sci., 1997, 39, 1981.

30 G. S. Frankel, J. Electrochem. Soc., 1998, 145, 2186.

31 R. Tunold, H. Holtan, M. B. H. Berge, A. Lasson and R. SteenHansen, Corros. Sci., 1977, 17, 353.

32 Y. Xin, K. Huo, H. Tao, G. Tang and P. K. Chu, Acta Biomater., 2008, 4, 2008. 